\title{
Cardiopatías en Canis familiaris en Lima Metropolitana
}

Cardiopathies in Canis familiaris in Metropolitan Lima

Fabrizio Gavazzo Mitrovic, Mv. Ursula Bezold ${ }^{1}$

http://dx.doi.org/10.21503/CienciayDesarrollo.2006.v6.05

\section{RESUMEN}

El desarrollo de la cultura por el bienestar animal y los nuevos avances de la tecnología han hecho que la clínica del día a día se conforme de nuevas técnicas y procedimientos que nos ayudan a combatir la interminable batalla contra los agentes causales de enfermedades, tanto reversibles como irreversibles.Entre estos males encontramos uno que hasta el momento había sido poco diagnosticado en nuestro medio y que cobra muchas vidas pasando desapercibido: las cardiopatías. Hoy en día son diagnosticadas por medio de exámenes complementarios, como el electrocardiograma y el ultrasonido. El objetivo de este estudio fue determinar el porcentaje de presentación de enfermedades cardiacas y cuál de estas era la que se encontraba con mayor frecuencia. Se controlaron 100 animales teniendo en cuenta la historia clínica, el examen físico, electrocardiografía y cuando se determinó necesario, se corrieron exámenes complementarios extras, como el ecocardiograma, análisis sanguíneos y rayos $x$. Del total de los 100 pacientes se encontró que 59 de ellos presentaban una variación en el trazado electrocardiográfico. Desde luego, no siempre fueron por problemas intrínsecos del corazón, pero al final el estudio reveló que de los problemas netamente cardiacos, las insuficiencias valvulares tomaban el primer lugar con $32,5 \%$, seguidas de los problemas del músculo cardiaco con un $8,5 \%$ y, con un porcentaje menor con $3.2 \%$ para las enfermedades congénitas, así como las parasitarias.

Palabras clave: cardiovascular, enfermedad, electrocardiograma, miocardio.

\section{ABSTRACT}

The development of a culture for the well being of animals and the new developments of technology have done that the day to day clinic approaches adopt new techniques and procedures which help us to fight the endless battle against the noxas that cause illnesses, reversible and otherwise. Among those we found one that few diagnosed in our environment takes a lot of lives being unnoticed: cardiopathies. Nowadays they are diagnosed through complementary exams such as electrocardiogram and ultrasound. The subject of the study was to establish the percentage of cardiac conditions and which of them were prevalent. One hundred animals were evaluated through anamnesis, auscultation, electrocardiography, and when it was necessary echocardiogram, blood tests and $x$ rays. Of the total of one hundred patients we found that 59 of them showed a variation on the drawings of the electrocardiogram. Of course, not always were problems intrinsic to the heart but at

1. Facultad de Ciencias Agropecuarias, Escuela Profesional de Medicina Veterinaria - Universidad Alas Peruanas. 
the end the study showed that a truly cardiac problem, valve insufficiency, takes the first place with $32,5 \%$, followed by those of the cardiac muscle with $8.5 \%$, and with a less percentage of $3,2 \%$ for congenital as well as parasitic illnesses.

\section{INTRODUCCIÓN}

En los últimos años, tanto en medicina humana así como en medicina veterinaria, se han determinado las diferentes enfermedades que acosan al corazón.

En países vecinos se ha señalado que las enfermedades que más sufre el canino son las insuficiencias valvulares, como por ejemplo la degeneración valvular mixomatosa (Belleremian y Mucha ${ }^{1}$ ). En este caso el mecanismo de acción parece ser la infiltración de mucopolisacáridos de la capa esponjosa hacia la fibrosa en la valva de la válvula (Fox et $\mathrm{al}^{2}$ ).

La enfermedad que le sigue en la escala de la prevalencia es la cardiomiopatía dilatada, la cual tiene una patogénesis algo incierta, ya que las teorías sobre esta enfermedad abundan (Tilley, 2001).

La falta de contracción del miocardio, la excesiva fibrosis y la subsecuente falta de gasto cardiaco, llevan al animal a la insuficiencia cardiaca congestiva (Mucha ${ }^{3}$ ).

Las enfermedades como efusión pericárdica, idiopaticas o no, se relacionan a traumatismos y el diagnostico mediante el electrocardiograma es sumamente fácil, debido a los complejos PQRST disminuidos en voltaje (Swift ${ }^{4}$.

Las enfermedades congénitas, con su baja prevalencia y un grado de dificultad sumamente alto para diagnosticarlas (Castacurta ${ }^{5}$ ) son un reto para el profesional.

Las afecciones que son acarreadas por parásitos como Dirofilaria immitis prevalecen en zonas aledañas a las riveras, por lo que se encontraron ci- fras significativas en San Martín de Porres, Rimac y Lima (Acuna ${ }^{6}$.

\section{MATERIAL Y MÉTODO}

Muestra: grupo de 100 caninos seleccionados previamente por el clínico por sospecha de cardiopatía en la ciudad de Lima Metropolitana.

Criterios de inclusión: Sexo, edad, peso y tipo de lesión.

Instrumentos: Estetoscopio, Ecocardiografo, Rayos $\mathrm{x}$, $\mathrm{y}$ laboratorio

\section{Procedimientos}

El protocolo del estudio fue la toma de datos, la auscultación y el electrocardiograma de los 100 pacientes.

Estos exámenes se llevaron a cabo en diversas clínicas veterinarias del sector de Lima metropolitana.

Los animales previamente auscultados fueron llevados a una mesa aislada de interferencias y en posición de cúbito lateral derecha se conectaron los cuatro electrodos a cada uno de los miembros, con el fin de realizar el trazado electrocardiográfico. Este se lee en sus dos coordenadas ( $x$ e y) con parámetros de milivoltios y segundos respectivamente. Tomando las respectivas medidas se puede determinar tanto el ritmo del corazón como la dimensión de sus cuatro cámaras y por consiguiente, saber cual de las válvulas está afectada.

En los animales en los cuales el diagnóstico no fue del todo seguro, se procedió hacer otras pruebas complementarias como los Rayos $\mathrm{x}$ y el 
Ultrasonido, para de esta forma, llegar a un diag- RESULTADOS nóstico definitivo.

Del total de 100 animales muestreados,

Los parámetros como peso, edad, sexo, 50 fueron hembras y 50 machos, de los cuales 41 y tipo de lesión se evaluaron en todo momento, animales estaban en perfectas condiciones y 59 ya que por ejemplo los animales con un peso me- presentaron un trazado alterado.

nor o igual de $19 \mathrm{Kg}$. están más predispuestos a insuficiencias valvulares y los de un peso igual o mayor de $20 \mathrm{Kg}$. son más propensos a las cardiomiopatías.

Tabla 1. Perros enfermos con cardiopatías de acuerdo a la edad

\begin{tabular}{ccc}
\hline Enfermos (años) & Nro. enfermos & $\%$ \\
\hline $0-1$ & 8 & 13,5 \\
$2-3$ & 9 & 15,25 \\
$4-5$ & 10 & 16,9 \\
$6-7$ & 10 & 16,9 \\
$8-9$ & 13 & 22 \\
$10-11$ & 3 & 5,08 \\
$12-13$ & 2 & 3,3 \\
$14-15$ & 2 & 3,3 \\
$16-17$ & 2 & 3,3 \\
Total & 59 & 100 \\
& & \\
\hline
\end{tabular}

Se muestra la cantidad total de los animales muestreados y la prevalencia que ocurre en un rango entre los 2 y 9 años.

Tabla 2. Perros enfermos con cardiopatías de acuerdo al peso

\begin{tabular}{|c|c|c|c|}
\hline Raza & Raza grande(> 20 kg) & Raza chica $(\leq 19 \mathrm{~kg})$ & Total \\
\hline Peso (kg) & 23 & 36 & 59 \\
\hline$\%$ & 38,9 & 61,1 & 100 \\
\hline
\end{tabular}

Se muestra que en cuanto al peso se encontró que los animales de más de $20 \mathrm{Kg}$., la cardiomiopatía dilatada prevalecía, a diferencia de los animales de igual o menor peso a $19 \mathrm{Kg}$. en donde las insuficiencias valvulares fueron el hallazgo más común.

De acuerdo a los estudios estadísticos (diferencia de proporciones, $\mathrm{P}<0,5$ ) se halló que los animales mas afectados se encuentran en el grupo de los de 19 o menos $\mathrm{kg}$ de peso. 
Tabla 3. Perros sanos y enfermos con cardiopatías de acuerdo al sexo

\begin{tabular}{lrrr}
\hline & Hembra & Macho & Total \\
& & & \\
\hline Sanos & 26 & 15 & 41 \\
Enfermos & 24 & 35 & 59 \\
Total & 50 & 50 & 100 \\
& & & \\
\hline
\end{tabular}

Se muestra que la variable del sexo determinó que los machos están mas predispuestos a enfermedades cardiacas que las hembras.

Tabla 4. Número de perros con cardiopatías según el sexo y la lesión

\begin{tabular}{ccccccccccccccccccc}
\hline Sexo & Arritmia & $\begin{array}{c}\text { Insuficien- } \\
\text { cia mitral }\end{array}$ & $\begin{array}{c}\text { Insufi- } \\
\text { ciencia } \\
\text { tricuspíde }\end{array}$ & $\begin{array}{c}\text { Insufic } \\
\text { au-vent }\end{array}$ & CMD & $\begin{array}{c}\text { Efusión } \\
\text { pericárdica }\end{array}$ & ESA & $\begin{array}{c}\text { Dirofila- } \\
\text { riasis }\end{array}$ & Total \\
\hline \multirow{3}{*}{ Hembra } & Nro & $\%$ & Nro & $\%$ & Nro & $\%$ & Nro & $\%$ & Nro & $\%$ & Nro & $\%$ & Nro & $\%$ & Nro & $\%$ & Nro & $\%$ \\
Macho & 15 & 62,5 & 2 & 8,3 & 4 & 16,6 & 0 & 0 & 2 & 3,3 & 1 & 4,1 & 0 & 0 & 0 & 0 & 24 & 40,6 \\
Total & 24 & 45,7 & 11 & 31,4 & 5 & 14,2 & 1 & 2,5 & 3 & 3,6 & 4 & 11,4 & 1 & 2,8 & 1 & 2,8 & 35 & 59,4 \\
\hline
\end{tabular}

CMD = Cardiomiopatia dilatada

ESA = Estenosis subaortica

Se muestra los diferentes tipos de lesión encontrada en el estudio y su relacionan al sexo de los pacien-

tes.

\section{DISCUSIÓN}

El objetivo del estudio fue demostrar el alto porcentaje de enfermedades cardiovasculares que existen en nuestro medio, ya que aún no se publican artículos referentes a estas patologías.

Podemos decir que hemos llegado a la conclusión de que existe un porcentaje mucho mayor de lo que se piensa en cuanto a cardiopatías y que nuestro estudio concuerda con otros autores en lo referente a sexo, edad y peso (Bellerenian \& Mucha').

De la misma forma no podemos concordar con el tipo de lesión, ya que en nuestro estudio se encontró un valor muy alto $(16,9 \%)$ de insuficiencias tricúspides (válvula derecha), lo que es raro, ya que esta válvula trabaja a una presión de tan solo $1 / 5$ de la válvula mitral (válvula izquierda), por lo que su degeneración no debería ser un hallazgo frecuente. Por consiguiente, en el momento nos encontramos haciendo nuevos estudios de la válvula previamente mencionada, mediante cortes histológicos, con el fin de determinar la noxa.

\section{REFERENCIAS BIBLIOGRÁFICAS}

1. Bellerenian, G., Mucha, C. Afecciones Cardiovasculares en Pequeños Animales. Buenos Aires: Intermedica 2001.

2. Fox, P. Cardiology. New Cork: Harcourt 1999.

3. Mucha, C., Aparecido, C., Bellerenian, G., Ynaraja, E. Atlas de Cardiología de Pequeños Animales. Buenos Aires: Intermedica 2004. 
4. Swift, S. Manual de Medicina y Cirugía Cardiorrespiratoria en Pequeños Animales. Madrid: Harcourt 2000.

5. Castacurta, L. Atlas do Coracao. Sao Paulo: Manole 1998.
6. Acuna, P. Determinación de la Prevalencia de Dirofilaria immitis en los distritos de San Martín de Porres, Rimac, Lima. Tesis para la licenciatura en Medicina Veterinaria. Lima: UNMSM 2003. 